\title{
Clinicopathologic and molecular characteristics of EGFR-mutant lung adenocarcinomas that transform to small cell lung cancer after TKI therapy
}

\author{
Li Yu ${ }^{1,2}$, Lyudmila Bazhenova ${ }^{2}$, Kathryn Gold², Lisa Tran², Van Hilburn², Peter Vu², Sandip Pravin Patel \\ ${ }^{1}$ Department of Oncology, Shengjing Hospital of China Medical University, Shenyang, China; ${ }^{2}$ Moores Cancer Center, University of California, San \\ Diego, CA, USA \\ Contributions: (I) Conception and design: L Yu, SP Patel; (II) Administrative support: L Yu, SP Patel; (III) Provision of study materials or patients: \\ All authors; (IV) Collection and assembly of data: L Yu, SP Patel; (V) Data analysis and interpretation: L Yu, SP Patel; (VI) Manuscript writing: All \\ authors; (VII) Final approval of manuscript: All authors. \\ Correspondence to: Sandip Pravin Patel, MD. Moores Cancer Center, University of California, San Diego, 3855 Health Sciences Drive, 92093 , La \\ Jolla, CA, USA. Email: patel@ucsd.edu.
}

Background: Small cell lung cancer (SCLC) transformation is one of the mechanisms of drug resistance to tyrosine kinase inhibitors (TKIs) in advanced epidermal growth factor receptor (EGFR)-mutated non-small cell lung cancer (NSCLC) and represents an increasingly recognized clinical dilemma.

Methods: We performed a retrospective review of 964 cases at the University of California, San Diego of patients with EGFR sensitizing mutations. Nine patients had a biopsy-confirmed small cell transformation. The unique gene alterations and clinicopathologic features were collected and analyzed.

Results: Nine cases (9/964, 0.9\%) were identified, all with stage IV adenocarcinoma (ADC) at diagnosis, 7 were poorly differentiated, and 7 had an EGFR exon 19 deletion. All nine patients had tumor protein p53 (TP53) mutation. Among seven cases that had next-generation sequencing (NGS), 5 harbored retinoblastoma 1 (RB1) loss. WNK lysine deficient protein kinase 1 (WNK1) mutation was found in two patients that had longer survival. The median time from the initial diagnosis to transformation was 22.7 months (IQR: 15.1-25.1). After small cell transformation on EGFR inhibition, all patients were treated with etoposide/ platinum, conferring a median progression-free survival (PFS) of 3.2 months (IQR, 2.2-6.5 months) and post-chemotherapy survival of 8.6 months (IQR, 4.0-19.0 months). Six patients, as they retained the initial EGFR mutations, resumed (did so after terminating chemotherapy)/continued (did so concomitantly with chemotherapy) TKIs with a median duration of 13.8 months (IQR, 3.8-27.7 months). Two patients received immunotherapy but had no benefit.

Conclusions: In our series, most patients with small cell transformation had poorly differentiated adenocarcinomas at baseline. RB1 loss was not universal in transformed patients in this series, though TP53 mutation was present in all tumor samples. WNK1 mutation may be a new resistance mechanism to TKIs that may be associated with improved survival.

Keywords: Lung adenocarcinoma; transformation; small cell lung cancer (SCLC); EGFR mutation; tyrosine kinase inhibitor (TKI)

Submitted Aug 20, 2021. Accepted for publication Nov 21, 2021.

doi: $10.21037 /$ tlcr-21-665

View this article at: https://dx.doi.org/10.21037/tlcr-21-665 


\section{Introduction}

Epidermal growth factor receptor (EGFR)-tyrosine kinase inhibitors (TKIs) play critical roles in the treatment of advanced EGFR mutated non-small cell lung cancer (NSCLC); however, drug resistance is expected, even with potent inhibitors such as Osimertinib (1). A variety of acquired resistance mechanisms have been reported, including secondary EGFR mutations, bypass or alternative pathway activation, and histologic transformation (2-4). Small cell lung cancer (SCLC) transformation is one of the mechanisms, accounting for $3 \%$ to $14 \%$ of all cases (3-6).

Although some previous studies reported that inactivation in tumor protein p53 (TP53) and retinoblastoma $1(R B 1)$ genes (7-9) might be associated with SCLC transformation of EGFR mutated NSCLC after TKIs therapy, the clinicopathologic characteristics and molecular mechanisms of such transformation remain largely unknown and need further investigation.

Here, we report 9 cases of SCLC transformed from $E G F R$-mutant lung adenocarcinoma (ADC) after EGFRTKIs treatment in a single institution during a 15 -year period. The primary objective of our study included analysis of the clinicopathologic features, epidemiology, treatment characteristics, and survival data of this group of cases. The secondary objective was to explore the unique gene alterations that contribute to SCLC transformation. We present the following article in accordance with the SRQR reporting checklist (available at https://tlcr.amegroups.com/ article/view/10.21037/tlcr-21-665/rc).

\section{Methods}

We performed a retrospective review of cases seen between August 1, 2005, and August 1, 2020, at Moores Cancer Center of the University of California, San Diego (UC San Diego). EMR (Epic systems corporation ${ }^{\circledR}$ ) was queried for lung cancer patients with EGFR-mutations who received EGFR TKIs therapy and etoposide-containing chemotherapy at some point during the disease course. Patients with a previous history of neuroendocrine tumors of the lung were excluded.

The study was conducted in accordance with the Declaration of Helsinki (as revised in 2013). The study was approved by the UC San Diego Institutional Review Board (No. HRPP\# 150348), and individual consent for this retrospective analysis was waived.

Out of 964 patients with EGFR mutations, 9 harbored small cell transformation. The SCLC transformation has been confirmed by the histologic diagnosis comprising immunohistochemistry (IHC) for neuroendocrine markers according to WHO classification guidelines of lung tumors, and the results of the genetic changes were summarized from genomic profiling in the EMR system. Duration of TKIs rechallenge, after small cell histologic transformation, was defined as the time from the date TKIs were restarted after etoposide/platinum chemotherapy to the date it was discontinued. The duration of TKIs continuation was the time from transformation to the date TKIs were discontinued. Post-chemotherapy survival was the time from the beginning of the etoposide/platinum to death, and progression-free survival (PFS) was the interval from the date when the etoposide/platinum treatment started until any progression event. Response assessment was based on the physician's evaluation of clinical and radiological data, documented in the EMR system, according to RECIST (Response Evaluation Criteria In Solid Tumors).

Next-generation sequencing (NGS) was performed using whole exon coverage of at least 73 genes in a CLIA (clinical laboratory improvement amendments)-certified laboratory. Matched "normal" sequencing was not available to further characterize germline or variant of undetermined significance (VUS), and thus bioinformatic tumor only approaches were utilized, and if question on nature of the mutation, PolyPhen-2 was utilized.

\section{Statistical analysis}

Data were presented as $\mathrm{n}(\%)$ for categorical variables and median (IQR) for continuous variables. Statistics were descriptive and retrospective, no formal prospective comparisons can be made. The bioinformatic analyses for NGS were performed by commercial vendors as part of their standard of care CLIA assay.

\section{Results}

\section{Patients' characteristics}

The clinicopathological, treatment and genomic details of patients were summarized in Tables 1,2. Nine cases of patients that transformed from EGFR-mutant NSCLC after TKIs (Gefitinib, Afatinib, Erlotinib, or Osimertinib) to SCLC were included. The median age was 60 years (IQR, $45-69$ years). There were 4 (44.4\%) males and 5 (55.6\%) females. Of all 9 patients, 4 were never smokers, and 5 were 


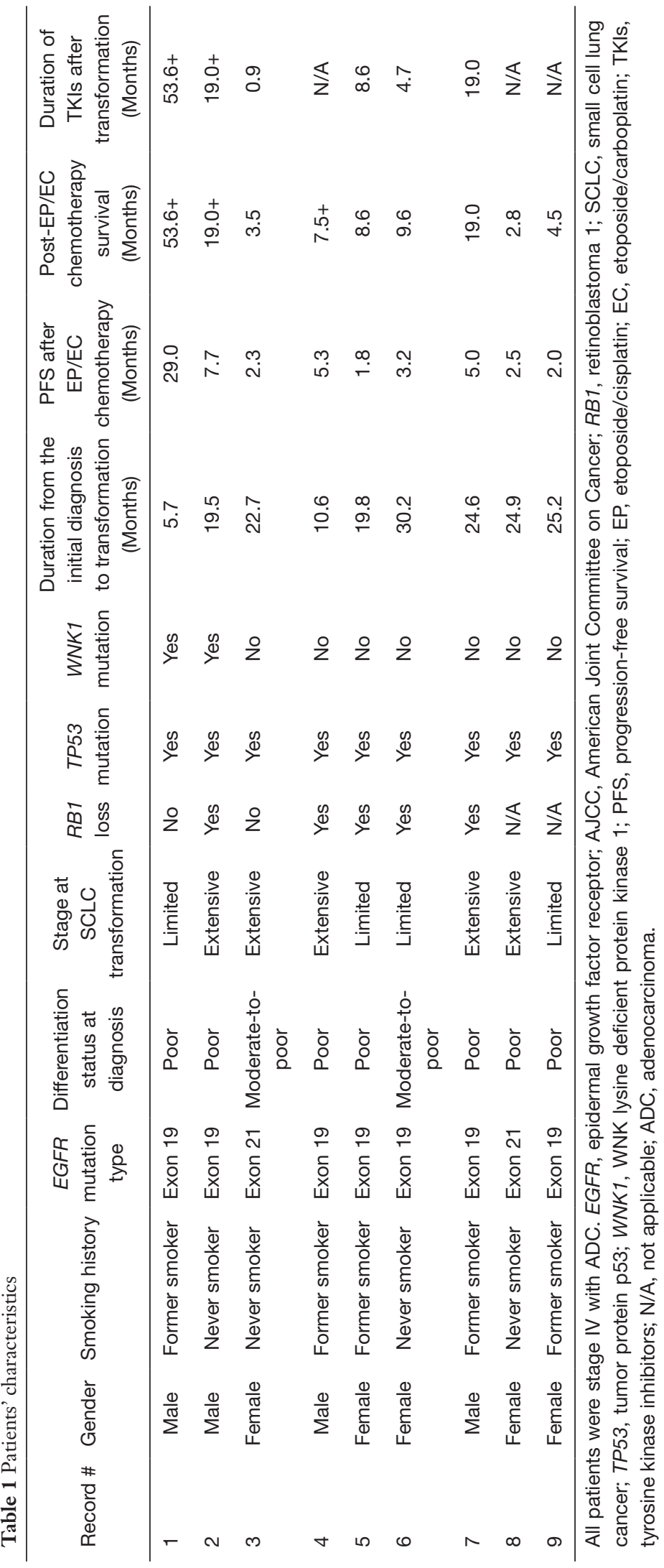


Table 2 EGFR TKIs patients received

\begin{tabular}{llll}
\hline Record \# & EGFR TKIs received before SCLC transformation & Rechallenge/continuation & EGFR TKIs received after SCLC transformation \\
\hline 1 & Gefitinib $\rightarrow$ Erlotinib & Continuation & Erlotinib $\rightarrow$ Osimertinib \\
2 & Afatinib & Continuation & Afatinib \\
3 & Erlotinib $\rightarrow$ Osimertinib & Rechallenge & Erlotinib \\
4 & Osimertinib & N/A & N/A \\
5 & Erlotinib $\rightarrow$ Osimertinib & Continuation & Osimertinib \\
6 & Afatinib & Rechallenge & Osimertinib \\
7 & Erlotinib $\rightarrow$ Osimertinib & Continuation & Osimertinib \\
8 & Erlotinib & N/A & N/A \\
9 & Erlotinib & N/A & N/A \\
\hline
\end{tabular}

EGFR TKIs that each patient received before and after SCLC transformation. Four patients' transformation occurred with respect to Osimertinib, and three of them received sequential treatment with 1st generation to 3rd generation of TKIs. EGFR, epidermal growth factor receptor; SCLC, small cell lung cancer; TKIs, tyrosine kinase inhibitors; N/A, not applicable.

former smokers. Two (22.2\%) patients had an exon 21-point mutation (L858R), and 7 (77.8\%) patients had an exon 19 deletion. All the 8 cases that had the molecular results after transformation retained the initial mutations (exon19del or L858R), one of the 9 cases lacked molecular testing after small cell transformation. The histological types of all the 9 patients were ADC, in which 7 patients were poorly differentiated at diagnosis, 2 were moderately-topoorly differentiated. All these patients were treated with at least one EGFR TKI before SCLC transformation as they were all at stage IV when initially diagnosed. The median time from the initial diagnosis to transformation was 22.7 months (IQR, 15.1-25.1 months).

\section{Post-transformation course}

After SCLC transformation, all patients were treated with etoposide combined with cisplatin or carboplatin (EP/EC) chemotherapy, conferring a median PFS of 3.2 months (IQR, 2.2-6.5 months) and post-chemotherapy survival of 8.6 months (IQR, 4.0-19.0 months). Four patients continued TKIs during EP/EC chemotherapy, and 2 patients resumed TKIs after EP/EC chemotherapy. The median duration of TKIs rechallenge/continuation after transformation was 13.8 months (IQR, 3.8-27.7 months) (Table 1).

\section{Molecular characteristics}

Nine patients had molecular test results. As shown in Tables 3-5, the patients had multiple co-mutations of the listed genes in their tumors. At baseline, 5 cases had NGS (comprehensive genome profile) results, 4 cases had targeted gene analysis. After transformation, 7 cases had NGS results, 1 case had targeted gene analysis, 1 case lacked molecular testing. As shown in Tables 1,3,4, all 9 patients had TP53 mutation. Among them, 6 patients had TP53 mutation both at baseline and after transformation. Two patients had TP53 mutation at baseline, while the mutation status was not available after transformation. One patient had TP53 mutation after transformation without mutation information at baseline. Seven cases had NGS results, among which, 5 cases had NGS both at baseline and after transformation. In these five cases, 4 cases had RB1 loss both at baseline and after transformation, but one case did not have RB1 loss. The other two patients had NGS after transformation, and one of them also harbored RB1 loss. There are two patients that had longer survival period (postchemotherapy survival $53.6 \mathrm{~m}+$ and $19.0 \mathrm{~m}+$, respectively). Interestingly, these two patients with longer survival period harbored a common unique genetic mutation for WNK lysine deficient protein kinase 1 (WNK1) (c.2567C >A p.T856K Missense variant and c.2176_2219delins (46) p. 
Table 3 The baseline gene mutations in the 7 patients that had NGS results

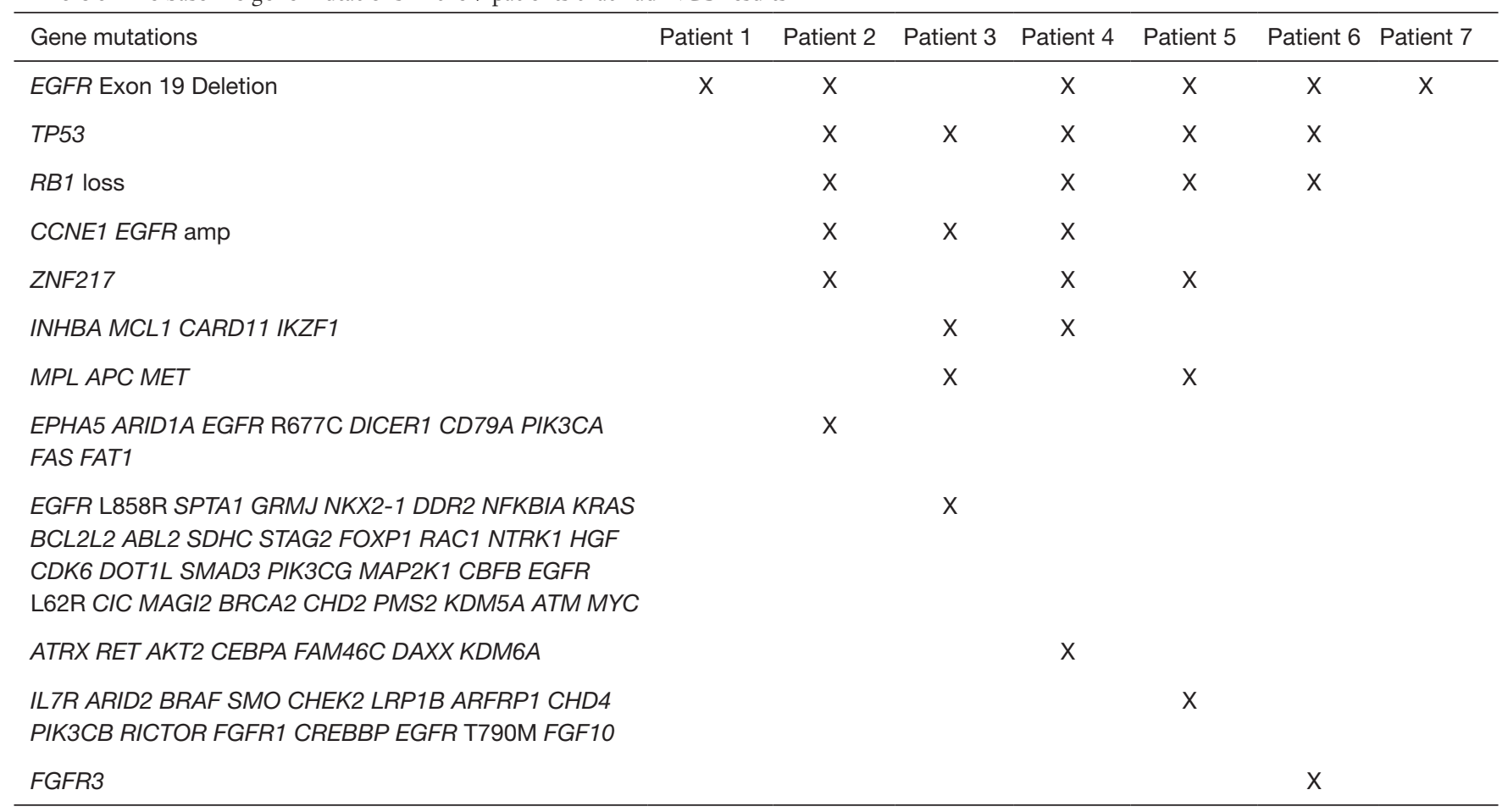

Patients 2-6 had NGS both at baseline and after transformation. Patients 1 and 7 had NGS after transformation. NGS, next-generation sequencing.

I726fs Frameshift, respectively). The $W N K 1$ gene mutation was acquired in one patient during treatment, but not clear if it was acquired in the other patient because this patient didn't have NGS at baseline.

\section{Discussion}

In our study, the histologic types of all 9 eligible patients were lung ADC that had been treated with at least one EGFR-TKI before SCLC transformation. Several studies have shown that alveolar type II cells, which were historically thought to be the origin of ADC, may be common precursors of both lung ADC and SCLC $(5,7,8,10)$. The majority $(77.8 \%)$ of our patients harbored tumors with poor differentiation at diagnosis, suggesting that patients with this feature may portend transformation to SCLC. In these transformed cases, it is unclear if they are transformed fully or partially as that would require a biopsy of each lesion, and many patients have dozens of lesions. Unfortunately, at present, there is no imaging test or other means to discern which lesion on a scan is NSCLC $v s$. transformed SCLC. In our cases, the median interval from the initial diagnosis of lung ADC to SCLC transformation was 22.7 months. In the cases previously reported, ranges of 10 months to 3 years have been showed $(4,6,9,11,12)$.

As shown in Table 1, seven out of nine SCLC transformations were observed in patients with $E G F R$ exon 19 mutations, and all the transformed SCLC retained the same EGFR mutation as the primary ADC. Similarly, previous studies revealed that SCLC transformation occurred more frequently in lung $\mathrm{ADC}$ with a deletion in EGFR exon 19 than in those with exon 21 L858R mutation, and the majority of patients retained the primary EGFR mutation indicating direct evolution from the original NSCLC (7-9,12).

There is no established guideline for treatment after the development of SCLC transformation. Treatment with platinum plus etoposide are standard therapies, and other strategies include irinotecan, taxanes, or radiotherapy (9). Some patients did respond well to these regimens $(9,13,14)$; but some cases received limited responses (15). The data of median post-chemotherapy survival from the transformation of two teams was 10.9 and 9 months, respectively $(9,12)$. Marcoux et al. showed a median PFS of 3.4 months after 
Table 4 The post-transformation gene mutations in the 7 patients that had NGS results

\begin{tabular}{|c|c|c|c|c|c|c|c|}
\hline Gene mutations & Patient 1 & Patient 2 & Patient 3 & Patient 4 & Patient 5 & Patient 6 & Patient 7 \\
\hline TP53 & $x$ & $x$ & $x$ & $\mathrm{X}$ & $x$ & $x$ & $\mathrm{X}$ \\
\hline RB1 loss & & $x$ & & $x$ & $x$ & $x$ & $x$ \\
\hline WNK1 & $x$ & $x$ & & & & & \\
\hline CCNE1 & & $x$ & $x$ & $x$ & & & \\
\hline KMT2C & & $x$ & & & & & $x$ \\
\hline$C D K 6$ & $x$ & & $x$ & & & & \\
\hline$N F 1$ & $x$ & & & & & & $x$ \\
\hline$M Y C$ & & & $x$ & & & & $X$ \\
\hline FGFR1 & & & & & $X$ & & $x$ \\
\hline $\begin{array}{l}\text { CDKN1B NOTCH1 KIF1B U2AF1L4 LRP1B ACVR1B TSC1 } \\
\text { CUL4B TET2 CUX1 MTRR KMT2D }\end{array}$ & $x$ & & & & & & \\
\hline $\begin{array}{l}\text { PIAS4 SRP68 TERT LMNA CBLB RNF139 PTPRT MALT1 } \\
\text { RB1 CFTR CRLF2 AMER1 TOP2A SLC47A2 BCLAF1 ZMYM3 } \\
\text { HSP90AA1 EGFR Copy number gain }\end{array}$ & & $x$ & & & & & \\
\hline $\begin{array}{l}\text { KIT NKX2-1 NFKBIA BCL2L2 EGFR L858R STAG2 FOXP1 MPL } \\
\text { RAC1 DOT1L SMAD3 EGFR L62R MET MAP2K1(MEK1) CIC } \\
\text { BRCA2 CHD2 PMS2 ATM }\end{array}$ & & & $x$ & & & & \\
\hline $\begin{array}{l}\text { KRAS CCND2 HRAS CCND1 PIK3CA GATA6 GPR124 AR } \\
\text { ARID1B ESR1 }\end{array}$ & & & & & & & $x$ \\
\hline
\end{tabular}

WNK1 mutation was found in both patients 1 and 2 that had longer survival period. VUS genes are not shown. NGS, next-generation sequencing; WNK1, WNK lysine deficient protein kinase 1; VUS, variant of undetermined significance.

platinum-etoposide chemotherapy (9). Their results are similar to our data: median PFS of 3.2 months and postchemotherapy survival of 9.6 months after the EC/EP treatment.

For patients with transformed SCLC, there are no guidelines about whether to restart/continue TKIs or not after/concomitantly with chemotherapy. In our study, 3 of the 9 cases showed CNS metastasis after SCLC transformation. Chemotherapy has poor penetration into the central nervous system (CNS), while Osimertinib is bioavailable and effective for CNS metastases, which is an issue in this patient population. Upon transformation, some researchers found, despite the retention of the $E G F R$-mutation, EGFR protein expression decreases, and patients have limited benefits from EGFR-TKIs $(7,16)$. However, Marcoux et al. (9) found 5/67 cases with EGFRamplification after SCLC transformation, in addition to the founder EGFR-mutation, suggesting that both EGFR- 
Table 5 The acquired gene mutations in the 5 patients (patient 2-6) that had NGS both at baseline and after transformation.

\begin{tabular}{|c|c|c|c|c|c|}
\hline Acquired gene mutations & Patient 2 & Patient 3 & Patient 4 & Patient 5 & Patient 6 \\
\hline KIT & & $\mathrm{X}$ & & & \\
\hline PPARG CD22 BCL6 & & & $\mathrm{x}$ & & \\
\hline RPTOR ABL2 IRS2 KLHL6 PDGFRB GNAS AURKA PTCH1 SMAD2 FLT3 KDM5A & & & & & $\mathrm{X}$ \\
\hline
\end{tabular}

NGS, next-generation sequencing.

downregulation and -upregulation can contribute to the unresponsiveness of transformed tumors to EGFR-TKIs. Whereas, our data showed that 3 out of 7 cases with NGS data exhibited $E G F R$-amplification at baseline (together with $C C N E$ mutation), while 1 patient displayed "EGFR copy number gain" after SCLC transformation (Tables 3,4). In our study, 2 patients resumed EGFR-TKIs (TKI rechallenge) at the completion of chemotherapy, and 4 patients continued TKIs therapy (TKI continuation) concomitantly with chemotherapy. The median treatment duration of TKIs after transformation was 13.8 months, which indicated that TKIs resumption/continuation might benefit such patients. Some cases also showed clinical benefits from re-challenging/continuing TKIs treatment $(9,14,17,18)$. Immunotherapy, which is disappointing in EGFR mutated NSCLC $(19,20)$, appears to be ineffective in the transformed SCLC (9). In our cases, 2 patients also had no clinical improvement after receiving immunotherapy, even though one patient had high tumor mutational burden (TMB) 15 Muts/Mb and programmed cell death-ligand 1 (PD-L1) tumor proportion score (TPS) $>50 \%$, which indicated that post-transformation, immunotherapy may not be effective.

Currently, the exact molecular changes associated with the transformation from lung ADC with EGFR mutations to SCLC after an initial response to EGFR TKIs remain largely unknown. Inactivation in TP53 and RB1 genes is the typical molecular feature for SCLC $(21,22)$, and both genes mutation were rarely observed in NSCLC (23). However, they were found usually altered in SCLC transformed patients $(7-9,22,24)$. In our study, we observed TP5 3 mutation in all the transformed patients and RB1 loss in 5 transformed patients. There were 2 cases without $R B 1$ loss, suggesting that RB1 loss is not universal in
SCLC transformed patients, similar to Marcoux's research results (9). Additionally, we noticed that 2 patients had longer survival period (post-chemotherapy survival 53.6+ and 19.0+ months, respectively). Of note, both patients harbored $W N K 1$ mutation. Interestingly, the cBioportal database (Figure 1) showed that $W N K 1$ altered in $1.25 \%$ to $30 \%$ of SCLC cases and only $2.32 \%$ to $6.09 \%$ of patients with lung ADC $(25,26)$. Previous research reported that WNK1 is a serine-threonine kinase that regulates ion transport across cell membranes, and influences PI3KAKT, TGF- $\beta$, and NF- $\mathrm{BB}$ signaling; WNK pathway has been implicated in numerous human diseases, including cancer (27). WNK1 Kinase's indirect effects on angiogenesis may play a role in promoting tumor growth and metastasis (28), though the specific role of WNK1 remains to be further studied. It is not clear whether WNK1 mutation is a contributor to SCLC transformation, or an enhancer of their prognosis. In this retrospective study, only 5 patients had NGS results before and after transformation. Optimally, the identical platform should be utilized pre and post-transformation. However, given the rare nature of the event and the limited number of patients, we felt if baseline changes portended transformation to SCLC (similar to $R B 1$ ), it would be important not to miss.

In summary, transformation to SCLC occurs in a small but important subset of patients with EGFR-mutant NSCLC that should be borne in mind when patients exhibit progression on TKIs, and re-biopsy is necessary. This single-institution study is limited by the small size of the cohort. In addition, the search strategy we used may make us unable to retrieve all the transformed patients. Because we designed our search to detect patients who received etoposide, we may have missed patients treated with alternative regimens. This may be why the proportion 


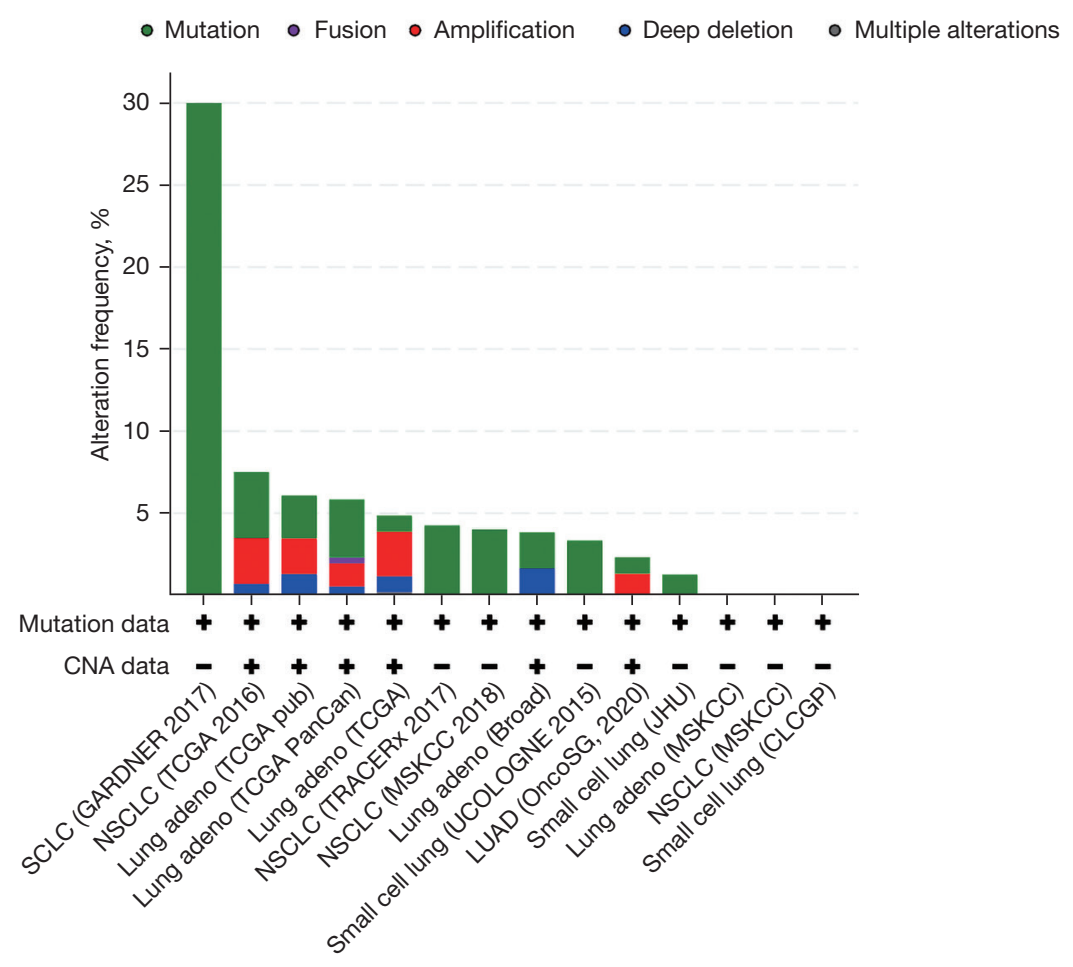

Figure 1 The gene alteration status of WNK1 in SCLC and NSCLC in the cBioportal database. WNK1 altered in 1.25-30\% of SCLC cases, and 2.32-6.09\% of patients with lung ADC. CNA, copy number alterations; Lung adeno, lung adenocarcinoma; SCLC, small cell lung cancer; NSCLC, non-small cell lung cancer; LUAD, lung adenocarcinoma; WNK1, WNK lysine deficient protein kinase 1; ADC, adenocarcinoma; TCGA, the cancer genome atlas; TRACERx, TRAcking cancer evolution through therapy (Rx); MSKCC, memorial Sloan-Kettering cancer center; JHU, Johns Hopkins university; CLCGP, clinical lung cancer genome project.

of transformed patients in our study is less than $1 \%$, a bit lower than other studies. Furthermore, variation in sequencing techniques was also a limitation of this study. Future research is required to characterize the features of these tumors in larger cohorts to seek optimal strategies, as the median PFS and post-chemotherapy survival after transformation is currently short. The underlying genomic changes that contribute to this drug-resistant mechanism require more detailed exploration to help predict histological SCLC transformation at earlier stages of the disease.

\section{Acknowledgments}

Funding: None.

\section{Footnote}

Reporting Checklist: The authors have completed the SRQR reporting checklist. Available at https://tlcr.amegroups.com/ article/view/10.21037/tlcr-21-665/rc

Peer Review File: Available at https://tlcr.amegroups.com/ article/view/10.21037/tlcr-21-665/prf

Conflicts of Interest: All authors have completed the ICMJE uniform disclosure form (available at https://tlcr.amegroups. com/article/view/10.21037/tlcr-21-665/coif). LY was partially supported by the foundation of "Plan of Cultivating Future Talents of Shengjing Hospital, China". LB is participating in DMC for ORIC and received honoraria for advisory boards for following companies: Genentech, Novartis, Boehringer Ingleheim, Bluprint, Takeda, Astra Zeneca, Bayer, G1 therapeutics, Beyondspring, Regeneron, Merck, Janssen, BMS, Daichi, Neuvogen, Turning point therapeutics and Epic sciences. KG's institution receives research support from Pfizer and Phamacyclics. KG receives consulting fees from: AstraZeneca, Takeda and Rakuten. 
SPP receives scientific advisory income from: Amgen, AstraZeneca, Bristol-Myers Squibb, Certis, Eli Lilly, Genentech, Illumina, Merck, Pfizer, Rakuten and Tempus. SPP's university receives research funding from: Amgen, AstraZeneca/MedImmune, Bristol-Myers Squibb, Eli Lilly, Fate Therapeutics, Genocea, Iovance, Merck, Pfizer and Roche/Genentech. The funders had no roles in the design and implementation of the study. The other authors have no conflicts of interest to declare.

Ethical Statement: The authors are accountable for all aspects of the work in ensuring that questions related to the accuracy or integrity of any part of the work are appropriately investigated and resolved. The study was conducted in accordance with the Declaration of Helsinki (as revised in 2013). The study was approved by the UC San Diego Institutional Review Board (No. HRPP\# 150348) and individual consent for this retrospective analysis was waived.

Open Access Statement: This is an Open Access article distributed in accordance with the Creative Commons Attribution-NonCommercial-NoDerivs 4.0 International License (CC BY-NC-ND 4.0), which permits the noncommercial replication and distribution of the article with the strict proviso that no changes or edits are made and the original work is properly cited (including links to both the formal publication through the relevant DOI and the license). See: https://creativecommons.org/licenses/by-nc-nd/4.0/.

\section{References}

1. Santoni-Rugiu E, Melchior LC, Urbanska EM, et al. Intrinsic resistance to EGFR-Tyrosine Kinase Inhibitors in EGFR-Mutant Non-Small Cell Lung Cancer: Differences and Similarities with Acquired Resistance. Cancers (Basel) 2019;11:923.

2. Blakely CM, Watkins TBK, $\mathrm{Wu} \mathrm{W}$, et al. Evolution and clinical impact of co-occurring genetic alterations in advanced-stage EGFR-mutant lung cancers. Nat Genet 2017;49:1693-704.

3. Yu HA, Arcila ME, Rekhtman N, et al. Analysis of tumor specimens at the time of acquired resistance to EGFR-TKI therapy in 155 patients with EGFR-mutant lung cancers. Clin Cancer Res 2013;19:2240-7.

4. Sequist LV, Waltman BA, Dias-Santagata D, et al. Genotypic and histological evolution of lung cancers acquiring resistance to EGFR inhibitors. Sci Transl Med 2011;3:75ra26.

5. Oser MG, Niederst MJ, Sequist LV, et al. Transformation from non-small-cell lung cancer to small-cell lung cancer: molecular drivers and cells of origin. Lancet Oncol 2015;16:e165-72.

6. Zakowski MF, Ladanyi M, Kris MG, et al. EGFR mutations in small-cell lung cancers in patients who have never smoked. N Engl J Med 2006;355:213-5.

7. Niederst MJ, Sequist LV, Poirier JT, et al. RB loss in resistant EGFR mutant lung adenocarcinomas that transform to small-cell lung cancer. Nat Commun 2015;6:6377.

8. Lee JK, Lee J, Kim S, et al. Clonal History and Genetic Predictors of Transformation Into Small-Cell Carcinomas From Lung Adenocarcinomas. J Clin Oncol 2017;35:3065-74.

9. Marcoux N, Gettinger SN, O'Kane G, et al. EGFRMutant Adenocarcinomas That Transform to Small-Cell Lung Cancer and Other Neuroendocrine Carcinomas: Clinical Outcomes. J Clin Oncol 2019;37:278-85.

10. Dorantes-Heredia R, Ruiz-Morales JM, Cano-García

F. Histopathological transformation to small-cell lung carcinoma in non-small cell lung carcinoma tumors. Transl Lung Cancer Res 2016;5:401-12.

11. Morinaga R, Okamoto I, Furuta K, et al. Sequential occurrence of non-small cell and small cell lung cancer with the same EGFR mutation. Lung Cancer 2007;58:411-3.

12. Ferrer L, Giaj Levra M, Brevet M, et al. A Brief Report of Transformation From NSCLC to SCLC: Molecular and Therapeutic Characteristics. J Thorac Oncol 2019;14:130-4.

13. Kim WJ, Kim S, Choi H, et al. Histological transformation from non-small cell to small cell lung carcinoma after treatment with epidermal growth factor receptor-tyrosine kinase inhibitor. Thorac Cancer 2015;6:800-4.

14. Kok VC, Lee CK, Chiang YH, et al. Extensive-Stage Small Cell Carcinoma Transformation From EGFR Del19-Mutant Lung Adenocarcinoma on Gefitinib at the Twelfth-Year Follow-Up Case Report. Front Oncol 2021;11:564799.

15. Roca E, Gurizzan C, Amoroso V, et al. Outcome of patients with lung adenocarcinoma with transformation to small-cell lung cancer following tyrosine kinase inhibitors treatment: A systematic review and pooled analysis. Cancer Treat Rev 2017;59:117-22.

16. Oxnard GR, Hu Y, Mileham KF, et al. Assessment of Resistance Mechanisms and Clinical Implications in 
Patients With EGFR T790M-Positive Lung Cancer and Acquired Resistance to Osimertinib. JAMA Oncol 2018;4:1527-34.

17. Lee S, Joo J, Kwak M, et al. Role of chemotherapy with epidermal growth factor receptor-tyrosine kinase inhibitor (EGFR-TKI) rechallenge in small cell transformation after EGFR-TKI failure: a case report. Onco Targets Ther 2018;11:3943-7.

18. Yang H, Liu L, Zhou C, et al. The clinicopathologic of pulmonary adenocarcinoma transformation to small cell lung cancer. Medicine (Baltimore) 2019;98:e14893.

19. Borghaei H, Paz-Ares L, Horn L, et al. Nivolumab versus Docetaxel in Advanced Nonsquamous Non-Small-Cell Lung Cancer. N Engl J Med 2015;373:1627-39.

20. Herbst RS, Baas P, Kim DW, et al. Pembrolizumab versus docetaxel for previously treated, PD-L1-positive, advanced non-small-cell lung cancer (KEYNOTE-010): a randomised controlled trial. Lancet 2016;387:1540-50.

21. Meuwissen R, Linn SC, Linnoila RI, et al. Induction of small cell lung cancer by somatic inactivation of both Trp53 and Rb1 in a conditional mouse model. Cancer Cell 2003;4:181-9.

Cite this article as: Yu L, Bazhenova L, Gold K, Tran L, Hilburn V, Vu P, Patel SP. Clinicopathologic and molecular characteristics of EGFR-mutant lung adenocarcinomas that transform to small cell lung cancer after TKI therapy. Transl Lung Cancer Res 2022;11(3):452-461. doi: 10.21037/tlcr-21665
22. George J, Lim JS, Jang SJ, et al. Comprehensive genomic profiles of small cell lung cancer. Nature 2015;524:47-53.

23. Cancer Genome Atlas Research Network. Comprehensive molecular profiling of lung adenocarcinoma. Nature 2014;511:543-50.

24. Offin M, Chan JM, Tenet M, et al. Concurrent RB1 and TP53 Alterations Define a Subset of EGFR-Mutant Lung Cancers at risk for Histologic Transformation and Inferior Clinical Outcomes. J Thorac Oncol 2019;14:1784-93.

25. Cerami E, Gao J, Dogrusoz U, et al. The cBio cancer genomics portal: an open platform for exploring multidimensional cancer genomics data. Cancer Discov 2012;2:401-4.

26. Gao J, Aksoy BA, Dogrusoz U, et al. Integrative analysis of complex cancer genomics and clinical profiles using the cBioPortal. Sci Signal 2013;6:pl1.

27. Gallolu Kankanamalage S, Karra AS, Cobb MH. WNK pathways in cancer signaling networks. Cell Commun Signal 2018;16:72.

28. Sie ZL, Li RY, Sampurna BP, et al. WNK1 Kinase Stimulates Angiogenesis to Promote Tumor Growth and Metastasis. Cancers (Basel) 2020;12:575. 